\title{
White Matter Integrity Deficit Associated with Betel Quid Dependence
}

\author{
Fulai Yuan ${ }^{1 \dagger}$, Xueling Zhu ${ }^{2,3 \dagger}$, Lingyu Kong ${ }^{3}$, Huaizhen Shen ${ }^{2}$, Weihua Liao ${ }^{3 *}$ \\ and Canhua Jiang ${ }^{4 *}$
}

${ }^{1}$ Health Management Center, Xiangya Hospital, Central South University, Changsha, China, ${ }^{2}$ School of Humanities and Social Sciences, National University of Defense Technology, Changsha, China, ${ }^{3}$ Department of Radiology, Xiangya Hospital, Central South University, Changsha, China, ${ }^{4}$ Department of Oral and Maxillofacial Surgery, Xiangya Hospital, Central South University, Changsha, China

OPEN ACCESS

Edited by:

Qinghua He,

Southwest University, China

Reviewed by:

Wei Liao,

University of Electronic

Science and Technology

of China, China

Yuan Zhong,

Nanjing Normal University,

China

*Correspondence:

Weihua Liao

369535642@qq.com;

Canhua Jiang

canhuaj@csu.edu.cn

tThese authors have contributed equally to this work.

Specialty section: This article was submitted to Psychopathology,

a section of the journal

Frontiers in Psychiatry

Received: 31 August 2017 Accepted: 25 September 2017 Published: 12 October 2017

Citation:

Yuan F, Zhu X, Kong L, Shen H, Liao W and Jiang C (2017) White Matter Integrity Deficit Associated with Betel Quid Dependence.

Front. Psychiatry 8:201. doi: 10.3389/fpsyt.2017.00201
Betel quid $(\mathrm{BQ})$ is a commonly consumed psychoactive substance, which has been regarded as a human carcinogen. Long-term BQ chewing may cause Diagnostic and Statistical Manual of Mental Disorders-IV dependence symptoms, which can lead to decreased cognitive functions, such as attention and inhibition control. Although betel quid dependence (BQD) individuals have been reported with altered brain structure and function, there is little evidence showing white matter microstructure alternation in BQD individuals. The present study aimed to investigate altered white matter microstructure in $\mathrm{BQD}$ individuals using diffusion tensor imaging. Tract-based spatial statistics was used to analyze the data. Compared with healthy controls, BQD individuals exhibited higher mean diffusivity (MD) in anterior thalamic radiation (ATR). Further analysis revealed that the ATR in BQD individuals showed less fractional anisotropy (FA) than that in healthy controls. Correlation analysis showed that both the increase of MD and reduction of FA in $B Q D$ individuals were associated with severity of $B Q$ dependence. These results suggested that $\mathrm{BQD}$ would disrupt the balance between prefrontal cortex and subcortical areas, causing declined inhibition control.

Keywords: betel quid dependence, diffusion tensor imaging, tract-based spatial statistics, fractional anisotropy, mean diffusivity, anterior thalamic radiation

\section{INTRODUCTION}

Betel quid (BQ), also known as betel nut or areca nut, is the fourth most commonly consumed psychoactive substance in the world (1). The World Health Organization regards BQ as a human carcinogen. It has been suggested that long-term BQ chewing can lead to betel quid dependence (BQD) according to the diagnostic criteria of substance dependence in Diagnostic and Statistical Manual of Mental Disorders-IV (DSM-IV), which can lead to decreased cognitive functions, such as attention and inhibition control (2-8). It is estimated that about 600 million people chew various types of BQ worldwide, predominantly in the countries of South and Southeast Asia (9). In Mainland China, BQ chewing is mainly practiced in Hunan and Hainan provinces (10).

Recently, BQD individuals have been reported with altered brain structure and function by use of various neuroimaging techniques (11-18). In the aspect of brain structure, our previous work with voxel-based morphormetry analysis suggested that $\mathrm{BQD}$ individuals showed less gray matter volume in dorsolateral prefrontal cortex, insula, ventral medial prefrontal cortex (VMPFC), 
and orbital frontal cortex (OFC) (18). For the brain function, another work with resting-state fMRI showed BQD individuals had decreased default mode network functional connectivity in VMPFC, OFC, and anterior cingulate cortex (14). Compared with other addictive substance, however, the neural networks and the pathophysiological mechanisms underlying BQ use remain unclear.

To date, there is little evidence examining white matter microstructure alternation in BQD individuals. A recent study using generalized q-sampling imaging reported increased diffusion anisotropy in ACC, parietal, temporal, and occipital regions in $\mathrm{BQ}$ chewers compared with healthy controls (12), which indicated that the influence of BQ chewing on the brain white matter. However, relatively small sample size of this study prevented us from drawing solid conclusion on white matter integrity alternation in BQ chewers.

The present study aimed to investigate the differences of white matter integrity between BQD individuals and healthy controls using diffusion tensor imaging (DTI). DTI is a noninvasive technique to study the brain microstructure and the integrity of the anatomical connectivity, which has been applied in studies of many types of addiction, including food (19-21), cocaine (22-24), alcohol (25), gambling (26), internet gaming (27). Fractional anisotropy (FA) and mean diffusivity (MD) are the most common indexes for measuring white matter integrity. Tract-based spatial statistics (TBSS) is a widespread method to process DTI data (28). For example, using TBSS, Romero et al. (22) found that cocaine-dependent subjects presented higher FA values in anterior cingulate and lower FA values in the anterior-posterior commissure plane. Many studies have suggested that there were similar brain mechanism for different types of addiction (29-31). Following this idea, we hypothesized that BQD individuals would show altered white matter compared with healthy controls. We also tried to explore if the FA/MD in certain brain regions could potentially predict the severity of BQ chewing. Understanding the neurobiology feature of BQ chewing would help us develop novel ways to diagnose and prevent BQ dependence.

\section{MATERIALS AND METHODS}

\section{Participants}

25 BQD individuals were recruited from the outpatient department of stomatology at Xiangya Hospital of Central South University. As reported before $(14,18)$, BQD individuals were diagnosed by a licensed MD level psychiatrist, according to the Structured Clinical Interview with DSM-IV criteria. Persons without use of BQ were defined as "healthy subjects," which were recruited through a combination of targeted site sampling, advertisement, and snowball sampling referrals. Participants were excluded if they met criteria for other substance dependence, had a medical history of any neurological or psychiatric disorder, or had claustrophobia/other disease preventing them from MRI scanning. The demographic and clinical characteristics of all participants had been reported in our previous study (18). This study was carried out in accordance with the recommendations of research ethical committee of Xiangya Hospital of Central South University of Hunan Province, Changsha, China. All subjects were given written informed consent in accordance with the Declaration of Helsinki. The protocol was approved by the Institutional Review Board at Xiangya Hospital of Central South University of Hunan Province, Changsha, China.

\section{Procedures}

25 BQD individuals finished the behavior interview. The detailed information on the behavioral interview was reported previously (18). Briefly, they finished the Betel Quid Dependence Scale (BQDS) to test their severity of BQ dependence (32). BQDS is a widely used scale for diagnosing BQD (32), which is a 16-item self-report instrument and gives scores for physical and psychological urgent need, increasing dose, and maladaptive use. The BQDS has an optimal cut-off score of 4, with the optimal sensitivity up to 0.926 , the specificity up to 0.977 , and the predictive accuracy up to $99.3 \%$. The BQDS exhibits high degrees of reliability and validity in both English-speaking and Chinese-speaking chewers $(32,33)$. All the BQD and healthy individuals finished the DTI scan.

\section{DTI Protocol}

All MRI images were acquired using a Siemens Skyra 3T scanner at Xiangya Hospital. Participants lay in the supine position on the scanner bed. Foam pads were used to minimize head motion. They were instructed to have a rest but keep their head very still during the DTI scan. The diffusion-tensor data for each subject was acquired using a diffusion-weighted, singleshot, spin-echo, echo-planar imaging sequence parallel to the line of the anterior-posterior commissure. The acquisition parameters were as follows: repetition time $=12,000 \mathrm{~ms}$, echo time $=72.4 \mathrm{~ms}$, matrix $=128 \times 128$, field of view $=256 \times 256$, slice thickness $=3 \mathrm{~mm}$, number of excitations $=2$, $b$-value $=1,000 \mathrm{~s} / \mathrm{mm}^{2}$, no gap, 50 axial slices, 64 directions. A dual spin-echo technique combined with bipolar gradients was employed to minimize the geometric distortion induced by eddy currents.

\section{TBSS Analysis}

The DTI data were processed by FMRIB's Diffusion Toolbox implemented in FSL (http://fsl.fmrib.ox.ac.uk). Diffusion data was corrected for eddy currents and possible head motion. Images were then skull-stripped (34), aligned to MNI space using FNIRT $(35,36)$, and resampled to $1 \mathrm{~mm}^{3}$. FA and MD were reconstructed by fitting a diffusion tensor model at each voxel. The voxelwise statistical analysis of the FA/MD data was carried out using TBSS (28), part of FSL. The mean FA image was created and thinned to create a mean FA skeleton that represented the centers of all tracts common to the group. Each subject's aligned FA/MD data were then projected onto this skeleton and the resulting data were fed into voxelwise crosssubject statistics. Finally, group differences of the resulting skeletonized FA/MD images were computed using non-parametric permutation methods [Randomize v2.1 in FSL (37)]. The null distribution at each voxel was constructed using 5,000 random 
permutations of the data. TFCE was used to correct for multiple comparisons across the whole brain. The mean FA/MD value in each significant cluster was then extracted using $f$ slmeants for each individual to do correlation analysis with BQDS. Robust regression was used for all correlation to minimize the impact of outliers using robustfit command in the MATLAB Statistics Toolbox.

\section{RESULTS}

Table 1 showed the demographic and clinical characteristics for BQD individuals and healthy controls. The two groups did not differ in terms of age $[t(48)=1.06, p=0.29]$, but the healthy control group was educated for longer time $[t(48)=-2.47$, $p=0.017]$. In later analysis, years of education was used as a covariate in the model.

TBSS results showed that, after controlling for education, there was no significant difference in FA between BQD individuals and healthy controls. BQD individuals showed higher $\mathrm{MD}$ in bilateral anterior thalamic radiation (ATR) than healthy controls (Table 2). The mean FA values within

TABLE 1 | Demographic and clinical characteristics of participants $(M \pm S D)$.

\begin{tabular}{lccc}
\hline & \multicolumn{1}{c}{$\begin{array}{c}\text { Betel } \\
\text { quid (BQ) } \\
\text { individuals }\end{array}$} & Controls & Statistics \\
& $29.87 \pm 4.71$ & $28.23 \pm 5.92$ & $t(48)=1.06, p=0.29$ \\
Age (years) & $14.39 \pm 5.19$ & $17.31 \pm 2.87$ & $t(48)=-2.47, p=0.017$ \\
$\begin{array}{l}\text { Education (years) } \\
\text { Betel Quid }\end{array}$ & $10.87 \pm 1.71$ & - & - \\
$\begin{array}{l}\text { Dependence Scale } \\
\text { Duration of BQ } \\
\text { chewing (years) }\end{array}$ & $12.96 \pm 5.05$ & - & - \\
$\begin{array}{l}\text { Dosage of BQ } \\
\text { chewing (g/day) }\end{array}$ & $48.48 \pm 17.54$ & - & - \\
\hline
\end{tabular}

TABLE 2 | Summary of tract-based spatial statistics (TBSS) results (Betel quid individuals > controls).

\begin{tabular}{lcccccc}
\hline \multirow{2}{*}{$\begin{array}{l}\text { Brain } \\
\text { region }\end{array}$} & $\begin{array}{c}\text { Cluster } \\
\text { size }\end{array}$ & \multicolumn{3}{c}{ MNI } & TFCE-corrected $\boldsymbol{p}$ \\
\cline { 3 - 5 } & & $\boldsymbol{x}$ & $\boldsymbol{y}$ & $\boldsymbol{z}$ & \\
\hline $\mathrm{R}$ & ATR & 272 & 3 & -5 & 10 & $<0.001$ \\
$\mathrm{~L}$ & ATR & 153 & -6 & -3 & -1 & $<0.001$ \\
\hline
\end{tabular}

L, left; R, right; MNI, Montreal Neurological Institute; TFCE, threshold-free cluster enhancement; ATR, anterior thalamic radiation.

The voxel size in TBSS analysis is $1 \mathrm{~mm} \times 1 \mathrm{~mm} \times 1 \mathrm{~mm}=1 \mathrm{~mm}^{3}$. these two clusters showing MD differences were extracted and compared between these two groups. As showed in Figure 1, results revealed that $\mathrm{BQD}$ individuals showed smaller FA values in bilateral ATR than healthy controls [left: $t(48)=2.85$, $p<0.01$, Cohen's $d=0.82$; right: $t(48)=2.93, p<0.01$, Cohen's $d=0.85]$. Correlation analysis showed that, in BQD group, the FA in bilateral ATR could predict the score in the BQDS [left: $r(25)=-0.628, p<0.001$, right: $r(25)=-0.637, p<0.001]$, and the MD in bilateral ATR could also predict the score in the BQDS [left: $r(25)=0.529, p=0.006$, right: $r(25)=0.534$, $p=0.006]$.

\section{DISCUSSION}

Comparing 25 BQD individuals with 25 healthy controls, the present study aimed to investigate altered white matter integrity associated with BQD individuals. Results suggested that BQD individuals showed smaller FA but larger MD in bilateral ATR than healthy controls. Moreover, these measures could potentially predict the severity of BQ dependence.

These results first emphasized the importance of the ATR in BQ dependence. The ATR is the radiation of fibers connecting the anterior and medial thalamic nuclei and the cerebral cortex of the frontal lobe via the anterior limb of the internal capsule (38). Altered white matter integrity has been revealed to be associated with major psychiatric disorders in previous investigation [see Ref. (39) for a review], such as depression (40-47), schizophrenia (48), and addiction (49). Especially, studies using DTI in different substance and behavioral addiction have examined addiction-related white matter integrity and reported consistent results in the ATR. For example, Bora and his colleagues found opiate addiction was associated with reduced FA in the ATR, corpus callosum, and longitudinal fasciculus (50). Reduced FA in the ATR and several brain regions was revealed to be related with obesity (21). In pathological gamblers, widespread lower white matter integrity was reported in multiple brain regions including the ATR (26). Furthermore, internet gaming disorder subjects exhibited abnormal FA values in the ATR (27). The pattern of white matter change in the ATR suggested the important role of the ATR in the neurobiology of addiction. Our study extended these prior findings by providing new evidence for abnormal white matter in the ATR in BQD individuals. In addition, we also observed $\mathrm{MD}$ increase with $\mathrm{BQ}$ dependence in the ATR. MD reflected the average diffusion across three dimensions, the increase of which was suggested

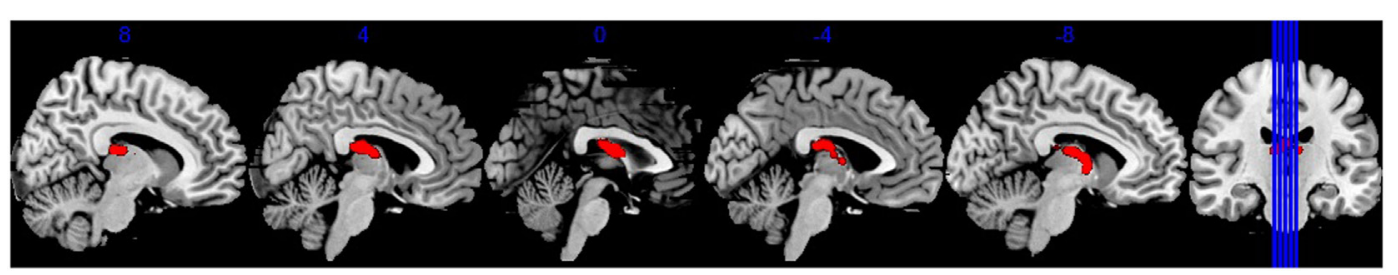

FIGURE 1 | Brain regions showed mean diffusivity difference between Betel quid individuals and controls. Figures are displayed in sagittal view. Numbers above each brain slices were the corresponding $\times$ value in the MNI space. 
to be associated with loss of neurons or myelin. However, few studies suggested MD changes associated with psychiatric disorders (51).

Considering the ATR connects the anterior and medial thalamic nuclei with the prefrontal cortex, the connection between these two neural systems allows us to control the basic impulses and allow more flexible pursuit of long-term goals (19, 52-54). The increased MD and decreased FA within the ATR could be explained as lacking of inhibition from prefrontal cortex to subcortical areas in BQ chewers (53). Similar mechanism has been suggested in both substance and behavioral addiction $(19,52-56)$, including hyperactivity in subcortical regions and hypoactivity in prefrontal regions $(29,43,57,58)$. Our results further showed that excessive BQ chewing would disrupt the balanced connection between these two neural systems, causing them to enjoy short-term "reinforcing effects" but ignore long-term negative consequences.

It should be noted that there were three limitations in current study. First, the study used a relatively small sample, and we didn't include female participants, which could potentially limit the generalization of our results to females. Second, the crosssection and correlation methods used in this study may limit our inference on the causality. Further longitudinal study should replicate and extend the conclusion from this study. Third, the conclusion was drawn basically on the structure connectivity, and further studies should try to combine DTI and functional connectivity to confirm our results.

\section{REFERENCES}

1. Boucher BJ, Mannan N. Metabolic effects of the consumption of Areca catechu. Addict Biol (2002) 7(1):103-10. doi:10.1080/13556210120091464

2. Chu NS. Neurological aspects of areca and betel chewing. Addict Biol (2002) 7(1):111-4. doi:10.1080/13556210120091473

3. Chen YJ, Chang JTC, Liao CT, Wang HM, Yen TC, Chiu CC, et al. Head and neck cancer in the betel quid chewing area: recent advances in molecular carcinogenesis. Cancer Sci (2008) 99(8):1507-14. doi:10.1111/ j.1349-7006.2008.00863.x

4. Lee C, Ko Y, Huang H, Chao Y, Tsai C, Shieh T, et al. The precancer risk of betel quid chewing, tobacco use and alcohol consumption in oral leukoplakia and oral submucous fibrosis in southern Taiwan. Br J Cancer (2003) 88(3):366-72. doi:10.1038/sj.bjc.6600727

5. Zain RB, Ikeda N, Gupta PC, Warnakulasuriya S, Wyk CW, Shrestha P, et al. Oral mucosal lesions associated with betel quid, areca nut and tobacco chewing habits: consensus from a workshop held in Kuala Lumpur, Malaysia, November 25-27, 1996. J Oral Pathol Med (1999) 28(1):1-4. doi:10.1111/ j.1600-0714.1999.tb01985.x

6. Gupta P, Ray C. Epidemiology of betel quid usage. Ann Acad Med Singapore (2004) 33(4 Suppl):31-6.

7. Ko YC, Chiang TA, Chang SJ, Hsieh SF. Prevalence of betel quid chewing habit in Taiwan and related sociodemographic factors. J Oral Pathol Med (1992) 21(6):261-4. doi:10.1111/j.1600-0714.1992.tb01007.x

8. Ko YC, Huang YL, Lee CH, Chen MJ, Lin LM, Tsai CC. Betel quid chewing, cigarette smoking and alcohol consumption related to oral cancer in Taiwan. J Oral Pathol Med (1995) 24(10):450-3. doi:10.1111/j.1600-0714.1995. tb01132.x

9. Gupta PC, Warnakulasuriya S. Global epidemiology of areca nut usage. Addict Biol (2002) 7(1):77-83. doi:10.1080/13556210020091437

10. Zhang SS, Li WH, Gao YJ, Liu ZW, Liu L, Tang JQ, et al. Betel-quid and oral submucous fibrosis: a cross-sectional study in Hunan province, China. J Oral Pathol Med (2012) 41(10):748-54. doi:10.1111/j.1600-0714. 2012.01166.x

\section{ETHICS STATEMENT}

This study was carried out in accordance with the recommendations of research ethical committee of Xiangya Hospital of Central South University of Hunan Province, Changsha, China. All subjects gave written informed consent in accordance with the Declaration of Helsinki. The protocol was approved by the Institutional Review Board at Xiangya Hospital of Central South University of Hunan Province, Changsha, China.

\section{AUTHOR CONTRIBUTIONS}

FY and XZ wrote the main manuscript text. WL and CJ conceived and designed the experiments. HS and LK conducted the experiments and collected data. FY and LK analyzed the results. All authors reviewed the manuscript.

\section{FUNDING}

This work was supported by research grants from the National Natural Science Foundation of China (grant number 81301211 to XZ), Foundation for the Author of National Excellent Doctoral Dissertation of PR China (grant number 201411 to XZ), Development and Reform Commission of Hunan Province (grant number 2016-No.65 to FY) and Introduction of Overseas Expertise and Intellectual Resources of Hunan Province (grant number 2016 to FY).

11. Chen F, Zhong Y, Zhang Z, Xu Q, Liu T, Pan M, et al. Gray matter abnormalities associated with betel quid dependence: a voxel-based morphometry study. Am J Transl Res (2015) 7(2):364-74.

12. Weng J-C, Kao T-W, Huang G-J, Tyan Y-S, Tseng H-C, Ho M-C. Evaluation of structural connectivity changes in betel-quid chewers using generalized q-sampling MRI. Psychopharmacology (2017) 234(13):1945-55. doi:10.1007/s00213-017-4602-0

13. Liu T, Li J, Zhang Z, Xu Q, Lu G, Huang S, et al. Altered long- and shortrange functional connectivity in patients with betel quid dependence: a resting-state functional MRI study. Cell Physiol Biochem (2016) 40(6):1626-36. doi:10.1159/000453212

14. Zhu X, Zhu Q, Jiang C, Shen $\mathrm{H}$, Wang F, Liao W, et al. Disrupted resting-state default mode network in betel quid-dependent individuals. Front Psychol (2017) 8:84. doi:10.3389/fpsyg.2017.00084

15. Liu T, Li J, Zhao Z, Zhong Y, Zhang Z, Xu Q, et al. Betel quid dependence is associated with functional connectivity changes of the anterior cingulate cortex: a resting-state fMRI study. J Transl Med (2016) 14(1):1-13. doi:10.1186/ s12967-016-0784-1

16. Huang X, Liu Z, Mwansisya TE, Pu W, Zhou L, Liu C, et al. Betel quid chewing alters functional connectivity in frontal and default networks: a resting-state fMRI study. J Mag Reson Imaging (2017) 45(1):157-66. doi:10.1002/jmri.25322

17. Liu T, Li J-J, Zhao Z-Y, Yang G-S, Pan M-J, Li C-Q, et al. Altered spontaneous brain activity in betel quid dependence: a resting-state functional magnetic resonance imaging study. Medicine (2016) 95(5):e2638. doi:10.1097/ MD.0000000000002638

18. Yuan F, Kong L, Zhu X, Jiang C, Fang C, Liao W. Altered gray-matter volumes associated with betel quid dependence. Front Psychiatry (2017) 8:139. doi:10.3389/fpsyt.2017.00139

19. He Q, Chen C, Dong Q, Xue G, Chen C, Lu Z, et al. Gray and white matter structures in the midcingulate cortex region contribute to body mass index in Chinese young adults. Brain Struct Funct (2015) 220(1):319-29. doi:10.1007/s00429-013-0657-9

20. Mueller K, Anwander A, Möller HE, Horstmann A, Lepsien J, Busse F, et al. Sex-dependent influences of obesity on cerebral white matter investigated 
by diffusion-tensor imaging. PLoS One (2011) 6(4):e18544. doi:10.1371/ journal.pone. 0018544

21. Papageorgiou I, Astrakas LG, Xydis V, Alexiou GA, Bargiotas P, Tzarouchi L, et al. Abnormalities of brain neural circuits related to obesity: a diffusion tensor imaging study. Mag Reson Imaging (2017) 37:116-21. doi:10.1016/j.mri.2016.11.018

22. Romero MJ, Asensio S, Palau C, Sanchez A, Romero FJ. Cocaine addiction: diffusion tensor imaging study of the inferior frontal and anterior cingulate white matter. Psychiatry Res (2010) 181(1):57-63. doi:10.1016/ j.pscychresns.2009.07.004

23. Ma L, Hasan KM, Steinberg JL, Narayana PA, Lane SD, Zuniga EA, et al. Diffusion tensor imaging in cocaine dependence: regional effects of cocaine on corpus callosum and effect of cocaine administration route. Drug Alcohol Depend (2009) 104(3):262-7. doi:10.1016/j.drugalcdep.2009. 05.020

24. Lane SD, Steinberg JL, Ma L, Hasan KM, Kramer LA, Zuniga EA, et al. Diffusion tensor imaging and decision making in cocaine dependence. PLoS One (2010) 5(7):e11591. doi:10.1371/journal.pone.0011591

25. Alhassoon OM, Sorg SF, Taylor MJ, Stephan RA, Schweinsburg BC, Stricker $\mathrm{NH}$, et al. Callosal white matter microstructural recovery in abstinent alcoholics: a longitudinal diffusion tensor imaging study. Alcohol Clin Exp Res (2012) 36(11):1922-31. doi:10.1111/j.1530-0277. 2012.01808.x

26. Joutsa J, Saunavaara J, Parkkola R, Niemela S, Kaasinen V. Extensive abnormality of brain white matter integrity in pathological gambling. Psychiatry Res (2011) 194(3):340-6. doi:10.1016/j.pscychresns.2011. 08.001

27. Jeong BS, Han DH, Kim SM, Lee SW, Renshaw PF. White matter connectivity and Internet gaming disorder. Addict Biol (2016) 21(3):732-42. doi:10.1111/ adb. 12246

28. Smith SM, Jenkinson M, Johansen-Berg H, Rueckert D, Nichols TE, Mackay CE, et al. Tract-based spatial statistics: voxelwise analysis of multisubject diffusion data. Neuroimage (2006) 31(4):1487-505. doi:10.1016/j. neuroimage.2006.02.024

29. Goldstein RZ, Volkow ND. Dysfunction of the prefrontal cortex in addiction: neuroimaging findings and clinical implications. Nat Rev Neurosci (2011) 12(11):652-69. doi:10.1038/nrn3119

30. Volkow ND, Wang G-J, Fowler JS, Tomasi D. Addiction circuitry in the human brain. Annu Rev Pharmacol Toxicol (2012) 52:321. doi:10.1146/ annurev-pharmtox-010611-134625

31. Volkow ND, Fowler JS. Addiction, a disease of compulsion and drive: involvement of the orbitofrontal cortex. Cereb Cortex (2000) 10(3):318-25. doi:10.1093/cercor/10.3.318

32. Lee C-Y, Chang C-S, Shieh T-Y, Chang Y-Y. Development and validation of a self-rating scale for betel quid chewers based on a male-prisoner population in Taiwan: the Betel Quid Dependence Scale. Drug Alcohol Depend (2012) 121(1):18-22. doi:10.1016/j.drugalcdep.2011.07.027

33. Herzog TA, Murphy KL, Little MA, Suguitan GS, Pokhrel P, Kawamoto CT. The Betel Quid Dependence Scale: replication and extension in a Guamanian sample. Drug Alcohol Depend (2014) 138:154-60. doi:10.1016/ j.drugalcdep.2014.02.022

34. Smith S. Fast robust automated brain extraction. Hum Brain Mapp (2002) 17(3):143-55. doi:10.1002/hbm.10062

35. Andersson JLR, Jenkinson M, Smith S. Non-linear registration, aka spatial normalisation. FMRIB Technical Report TR07JA2 (2007). Available from: https://www.fmrib.ox.ac.uk/datasets/techrep/

36. Andersson JLR, Jenkinson M, Smith S. Non-linear optimisation. FMRIB Technical Report TR07JA1 (2007). Available from: https://www.fmrib.ox. ac.uk/datasets/techrep/

37. Nichols T, Holmes A. Nonparametric permutation tests for functional neuroimaging: a primer with examples. Hum Brain Mapp (2002) 15(1):1-25. doi:10.1002/hbm.1058

38. Sprooten E, Lymer GK, Munoz Maniega S, McKirdy J, Clayden JD, Bastin ME, et al. The relationship of anterior thalamic radiation integrity to psychosis risk associated neuregulin-1 variants. Mol Psychiatry (2009) 14(3):237-8. doi:10.1038/mp.2008.136

39. Tekin S, Cummings JL. Frontal-subcortical neuronal circuits and clinical neuropsychiatry: an update. J Psychosom Res (2002) 53(2):647-54. doi:10.1016/ S0022-3999(02)00428-2
40. Coenen VA, Panksepp J, Hurwitz TA, Urbach H, Mädler B. Human medial forebrain bundle (MFB) and anterior thalamic radiation (ATR): imaging of two major subcortical pathways and the dynamic balance of opposite affects in understanding depression. J Neuropsychiatry Clin Neurosci (2012) 24(2):223-36. doi:10.1176/appi.neuropsych.11080180

41. Mark VH, Barry H, McLARDY T, Ervin FR. The destruction of both anterior thalamic nuclei in a patient with intractable agitated depression. J Nerv Ment Dis (1970) 150(4):266-72. doi:10.1097/00005053-19700400000002

42. Zhu X, Wang X, Xiao J, Liao J, Zhong M, Wang W, et al. Evidence of a dissociation pattern in resting-state default mode network connectivity in firstepisode, treatment-naive major depression patients. Biol Psychiatry (2012) 71 (7):611-7. doi:10.1016/j.biopsych.2011.10.035

43. Zhu X, Zhu Q, Shen H, Liao W, Yuan F. Rumination and default mode network subsystems connectivity in first-episode, drug-naive young patients with major depressive disorder. Sci Rep (2017) 7:43105. doi:10.1038/ srep43105

44. Zhu X, Wang X, Xiao J, Zhong M, Liao J, Yao S. Altered white matter integrity in first-episode, treatment-naive young adults with major depressive disorder: a tract-based spatial statistics study. Brain Res (2011) 1369:223-9. doi:10.1016/j.brainres.2010.10.104

45. Chen Y, Wang C, Zhu X, Tan Y, Zhong Y. Aberrant connectivity within the default mode network in first-episode, treatment-naive major depressive disorder. JAffect Disord (2015) 183:49-56. doi:10.1016/j.jad.2015. 04.052

46. Zhang X, Zhu X, Wang X, Zhu X, Zhong M, Yi J, et al. First-episode medication-naive major depressive disorder is associated with altered resting brain function in the affective network. PLoS One (2014) 9(1):e85241. doi:10.1371/journal.pone.0085241

47. Zhang X, Yao S, Zhu X, Wang X, Zhu X, Zhong M. Gray matter volume abnormalities in individuals with cognitive vulnerability to depression: a voxel-based morphometry study. J Affect Disord (2012) 136(3):443-52. doi:10.1016/j.jad.2011.11.005

48. Mamah D, Conturo TE, Harms MP, Akbudak E, Wang L, McMichael AR, et al. Anterior thalamic radiation integrity in schizophrenia: a diffusiontensor imaging study. Psychiatry Res (2010) 183(2):144-50. doi:10.1016/ j.pscychresns.2010.04.013

49. Mechtcheriakov S, Brenneis C, Egger K, Koppelstaetter F, Schocke M, Marksteiner J.A widespread distinct pattern of cerebral atrophy in patients with alcohol addiction revealed by voxel-based morphometry. J Neurol Neurosurg Psychiatry (2007) 78(6):610-4. doi:10.1136/jnnp.2006. 095869

50. Bora E, Yücel M, Fornito A, Pantelis C, Harrison BJ, Cocchi L, et al White matter microstructure in opiate addiction. Addict Biol (2012) 17(1):141-8. doi:10.1111/j.1369-1600.2010.00266.x

51. Sexton CE, Mackay CE, Ebmeier KP. A systematic review of diffusion tensor imaging studies in affective disorders. Biol Psychiatry (2009) 66(9):814-23. doi:10.1016/j.biopsych.2009.05.024

52. He Q, Xiao L, Xue G, Wong S, Ames SL, Bechara A. Altered dynamics between neural systems sub-serving decisions for unhealthy food. Front Neurosci (2014) 8:350. doi:10.3389/fnins.2014.00350

53. Noël X, Brevers D, Bechara A. A neurocognitive approach to understanding the neurobiology of addiction. Curr Opin Neurobiol (2013) 23(4):632-8. doi:10.1016/j.conb.2013.01.018

54. He Q, Xiao L, Xue G, Wong S, Ames SL, Schembre SM, et al. Poor ability to resist tempting calorie rich food is linked to altered balance between neural systems involved in urge and self-control. Nutr J (2014) 13(1):92. doi:10.1186/1475-2891-13-92

55. He Q, Chen M, Chen C, Xue G, Feng T, Bechara A. Anodal stimulation of the left DLPFC increases IGT scores and decreases delay discounting rate in healthy males. Front Psychol (2016) 7:1421. doi:10.3389/fpsyg.2016. 01421

56. He Q, Turel O, Bechara A. Brain anatomy alterations associated with Social Networking Site (SNS) addiction. Sci Rep (2017) 7:45064. doi:10.1038/ srep45064

57. Feil J, Sheppard D, Fitzgerald PB, Yücel M, Lubman DI, Bradshaw JL. Addiction, compulsive drug seeking, and the role of frontostriatal mechanisms in regulating inhibitory control. Neurosci Biobehav Rev (2010) 35(2):248-75. doi:10.1016/j.neubiorev.2010.03.001 
58. Volkow ND, Wang G-J, Fowler JS, Tomasi D, Telang F. Addiction: beyond dopamine reward circuitry. Proc Natl Acad Sci U S A (2011) 108(37):15037-42. doi:10.1073/pnas.1010654108

Conflict of Interest Statement: The authors declare that the research was conducted in the absence of any commercial or financial relationships that could be construed as a potential conflict of interest.
Copyright () 2017 Yuan, Zhu, Kong, Shen, Liao and Jiang. This is an open-access article distributed under the terms of the Creative Commons Attribution License (CC BY). The use, distribution or reproduction in other forums is permitted, provided the original author(s) or licensor are credited and that the original publication in this journal is cited, in accordance with accepted academic practice. No use, distribution or reproduction is permitted which does not comply with these terms. 